\title{
Matrix metalloproteinases and their inhibitors in gastric cancer
}

G I Murray, M E Duncan, E Arbuckle, W T Melvin, J E Fothergill

\begin{abstract}
Background-The matrix metalloproteinases (MMPs) and tissue inhibitors of matrix metalloproteinases (TIMPs) are strongly implicated in tumour invasion and metastasis.

Aims-To investigate the presence of individual MMPs and TIMPs in gastric cancer.

Methods-The presence of MMP-1, MMP-2, MMP-3, MMP-9, TIMP-1, and TIMP-2 was identified in a group of gastric cancers $(n=74)$ by immunohistochemistry using monoclonal antibodies. These antibodies were effective on formalin fixed, paraffin wax embedded sections. Results-A large proportion (94\%) of gastric cancers contained MMP-2; MMP-1 and MMP-9 were also detected in $73 \%$ and $70 \%$ of tumours respectively. MMP-3 was only present in $27 \%$ of tumours. MMP-1 and MMP-9 were found predominantly in intestinal type tumours. TIMP-1 and TIMP-2 were identified in $41 \%$ and $57 \%$ of tumours respectively. Immunoreactivity for individual MMPs or TIMPs was not identified in normal stomach.

Conclusions-This study shows the presence of matrix metalloproteinases, particularly MMP-2, and TIMPs in stomach cancer. Antibodies which are effective in formalin fixed, paraffin wax embedded sections are useful for the identification of MMPs and TIMPs in diagnostic specimens.
\end{abstract}

(Gut 1998;43:791-797)

Keywords: immunohistochemistry; matrix metalloproteinase; neoplasm; stomach

Department of Pathology G I Murray

E Arbuckle

Department of Molecular and Cell Biology, University of Aberdeen, UK $M$ E Duncan W T Melvin

J E Fothergill

Correspondence to: Dr G I Murray, Department of Pathology, University of Aberdeen, Foresterhill, Aberdeen AB25 2ZD, UK

Accepted for publication 15 June 1998

Cancer of the stomach is one of the commonest malignant tumours of the alimentary tract and is characterised by late clinical presentation, rapid progression, and poor survival. ${ }^{1}$ The reason for this poor prognosis is that, at the time of diagnosis, stomach cancer usually shows extensive local tumour invasion and frequent spread to metastatic sites, particularly lymph nodes. Spread of malignant tumours is a multistep process and many of the stages of tumour invasion require degradation or breakdown of the extracellular matrix and connective tissue surrounding tumour cells. ${ }^{2}{ }^{3}$ The matrix metalloproteinases (MMPs) are a family of zinc containing enzymes ${ }^{45}$ which are involved in the degradation of different components of the extracellular matrix, and there is considerable evidence to indicate that indi- vidual MMPs have important roles in tumour invasion and tumour spread..$^{5-9}$

The MMPs have been classified into collagenases, gelatinases, and stromelysins based on the in vitro substrate specificity of individual MMPs. ${ }^{40}$ These MMPs are multidomain proteins and are secreted as inactive precursors which are activated by cleavage of an $\mathrm{N}$ terminal propeptide. The gelatinases, particularly MMP-2, seem to be important in initial stages of tumour invasion ${ }^{11}$ as they degrade components of the basement membrane, while other MMPs contribute to the later stages of tumour invasion. ${ }^{5}$ More recently, several membrane bound MMPs have been identified in tumour cells. They have been designated membrane type $\mathrm{MMPs}^{12}{ }^{13}$ and are involved in the activation of MMP-2.

Tissue inhibitors of matrix metalloproteinases (TIMPs) are the major natural inhibitors of MMPs and several different TIMPs (TIMP-1 to TIMP-4) have been identified. ${ }^{14-17}$ The TIMPs are secreted proteins which complex with MMPs and are involved in regulating the activity and activation of individual MMPs. ${ }^{18}$ They are a homologous family of proteins containing two domains. The $x$ ray structure of a complex between TIMP-1 and the catalytic domain of MMP-3 has recently been reported. ${ }^{19}$ In vitro TIMP-2 has been shown to be capable of inhibiting tumour invasion. ${ }^{20}$

In this study we have investigated the presence of MMP-1 (interstitial collagenase), MMP-2 (72 $\mathrm{kDa}$ gelatinase), MMP-3 (stromelysin-1), MMP-9 (92 kD gelatinase), TIMP-1, and TIMP-2 in stomach cancer.

\section{Methods}

TISSUE

Samples of gastric cancer $(n=74)$ were obtained from gastrectomy specimens from patients (age range 41-87 years, 49 males, 25 females) who had undergone surgery for gastric cancer. All the patients had survived for at least one month following surgery and were followed up for between six and 36 months. The median survival was 16 months (95\% confidence interval 13-19 months). By the end of the follow up period there had been 37 (50\%) deaths. The specimens had been submitted to the Department of Pathology, University of Aberdeen, and had been fixed in formalin and embedded in paraffin wax. For this study a single block was selected from each case to include normal and tumour tissue. Histologically all the tumours were adenocarcinomas and there were $47(64 \%)$ intestinal type tumours and $27(36 \%)$ which were classified as 


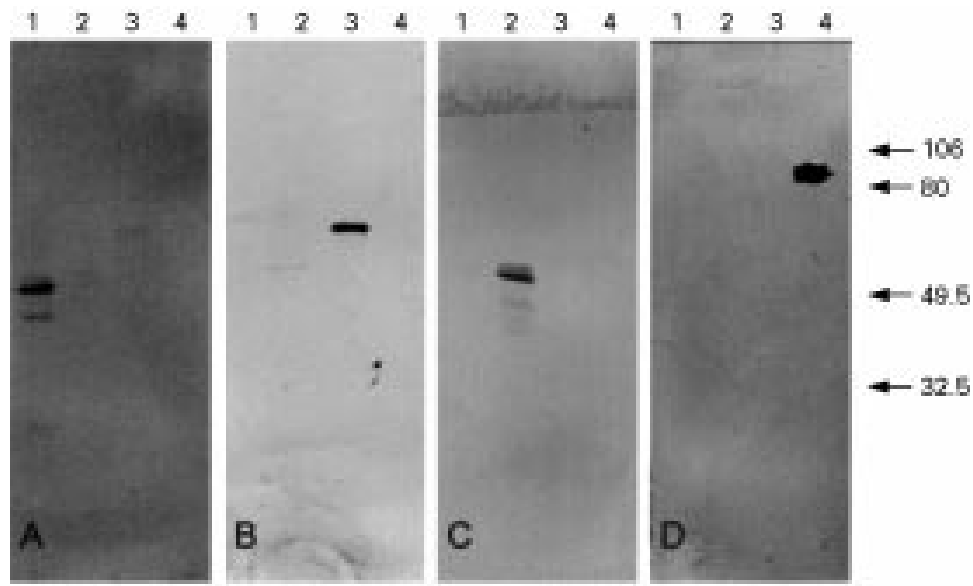

Figure 1 Immunoblot showing the specificity of the $M M P-1, M M P-2, M M P-3$, and $M M P-9$ antibodies. The membrane was immunostained with $M M P-1(A), M M P-2(B)$, $M M P-3(C)$, and MMP-9 antibody (D). Lane 1, MMP-1; lane 2, MMP-3; lane 3, MMP-2; lane 4, MMP-9. Molecular weight markers are shown on the right in kDa.
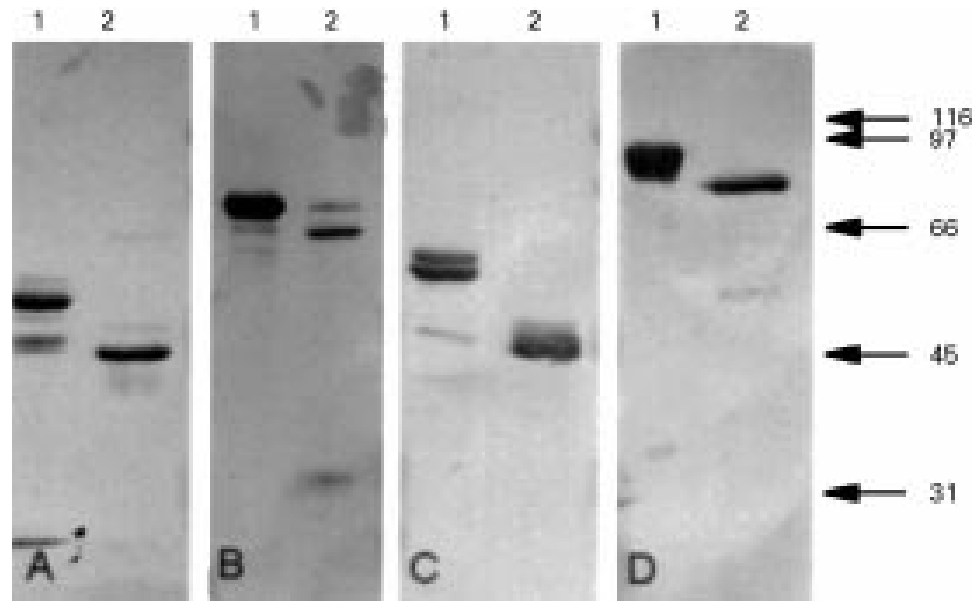

Figure 2 Immunoblots showing the reaction of the $M M P-1, M M P-2, M M P-3$, and $M M P-9$ monoclonal antibodies with the proenzyme and activated forms of the corresponding $M M P$. The membrane was immunostained with $M M P-1(A), M M P-2$ (B), MMP-3 (C), and MMP-9 antibody (D). Lane 1 contained the proenzyme and lane 2 the activated form of the MMP corresponding to the monoclonal antibody used for the immunostaining. Molecular weight markers are shown on the right in $k D a$.

diffuse tumours. Lymph node metastases were identified in $48(65 \%)$ cases while $26(35 \%)$ were free of lymph node metastases. TNM staging of the tumours showed that five were stage $1 \mathrm{a}, 17$ stage $1 \mathrm{~b}, 43$ stage 2 , eight stage $3 \mathrm{a}$, and one stage 4 . Poor survival correlated with increasing tumour stage $(\mathrm{p}=0.03)$ and the presence of lymph node metastases $(\mathrm{p}=0.02)$.

MONOCLONAL ANTIBODIES

Monoclonal antibodies to MMP-1, MMP-2, MMP-3, and MMP-9 were produced using synthetic peptides of length 11-13 residues, corresponding to unique sequences present in MMP-1, MMP-2, MMP-3, or MMP-9. The procedures used for the selection of peptides from the sequences of MMP-2, MMP-3, and MMP-9 were similar to those used to select the peptide for the development of the monoclonal antibody to MMP-1. ${ }^{21}$ The development and characterisation of the MMP-2 and MMP-9 monoclonal antibodies has recently been described. ${ }^{22}$ The amino acid sequences used were: SSFGFPRTVKH (MMP-1), TSLGLPPDVQRVD (MMP-2), MMP-3
(KSLRKLEPELH), and KLGLGADVAQVT (MMP-9). They corresponded to sequences on external loops in the $\mathrm{C}$ terminal domains of the MMPs. The peptides were synthesised at the Krebs Institute, University of Sheffield, Sheffield, and were checked for accuracy of synthesis by amino acid sequence analysis and mass spectrometry in the Protein Facility, University of Aberdeen. The synthetic peptides were conjugated to carrier proteins through an $\mathrm{N}$ terminal cysteine residue by $m$-maleimidobenzoic acid $N$-hydroxysuccinimide ester $(\mathrm{MBS})^{23}$ in the case of MMP-1 and MMP-3, and by glutaraldehyde ${ }^{24}$ for MMP-2 and MMP-9, and used as immunogens for the production of monoclonal antibodies as described for MMP-1. ${ }^{21}$ The peptides, linked to bovine serum albumin (BSA) by the above methods, were used for initial antibody screening. The BSA conjugates were bound to an enzyme linked immunosorbent asay (ELISA) plate by incubation overnight at $4^{\circ} \mathrm{C}$ in $50 \mathrm{mM}$ sodium carbonate/bicarbonate buffer, $\mathrm{pH} 9.6$, and the ELISA was performed as described previously. $^{25}$

The specificity of the monoclonal antibodies was shown by immunoblotting against purified MMP proenzymes. Individual MMPs $(0.4 \mu \mathrm{g}$ purified protein per lane) were subjected to sodium dodecyl sulphate polyacrylamide gel electrophoresis (SDS-PAGE) and then electrophoretically transferred to polyvinylene difluoride (PVDF) membrane. The membrane was then divided into four sections and each section incubated with one of the monoclonal antibodies. Antibody which had reacted with antigen on the membrane was detected using alkaline phosphatase conjugated goat antimouse IgG (Fc specific) with the substrate bromochloro-indolyl-phosphate/nitro blue tetrazolium (BCIP/NBT; Sigma, Poole, Dorset).

In order to investigate the reaction of the monoclonal antibodies with the activated forms of the MMPs, the proenzymes were activated in vitro. MMP-1 proenzyme was incubated with trypsin (L-1-tosylamide-2phenylethyl chloromethyl ketone treated) at a final concentration of $10 \mu \mathrm{g} / \mathrm{ml}$ in the presence of pro-MMP-3 at 0.75 of the molar concentration of pro-MMP-1 for 15 minutes at $37^{\circ} \mathrm{C}$. MMP-2 was activated by treatment with 4-aminophenyl mercuric acetate (APMA) at a final concentration of $1 \mathrm{mM}$ for one hour at $37^{\circ} \mathrm{C}$. MMP-3 and MMP-9 were activated by incubation with trypsin at a final concentration of $10 \mu \mathrm{g} / \mathrm{ml}$ for 15 minutes and two hours, respectively, at $37^{\circ} \mathrm{C}$. In all cases the activation reactions were terminated by boiling in SDS treatment buffer $(0.063 \mathrm{M}$ Tris, $\mathrm{pH} 6.8,2 \%$ SDS, $10 \%$ vol $/ \mathrm{vol}$ glycerol, $5 \% \mathrm{wt} / \mathrm{vol}$ 2-mercaptoethanol). A $0.6 \mu \mathrm{g}$ aliquot of protein (proenzyme or activated enzyme) was applied per lane prior to analysis by immunoblotting as described previously. Confirmation of activation of MMPs was obtained by determining sequences by Edman degradation following SDS-PAGE and transfer to PVDF membrane, ${ }^{26}$ and comparison with established amino acid sequences. ${ }^{27}$ 
Table 1 The presence of individual matrix metalloproteinases and tissue inhibitors of matrix metalloproteinases in different histological types of gastric cancer

\begin{tabular}{|c|c|c|c|}
\hline & Diffuse type $(n=27)$ & Intestinal type $(n=47)$ & All tumours $(n=74)$ \\
\hline \multicolumn{4}{|l|}{ MMP-1 } \\
\hline Strong & $6(22)$ & $22(47)$ & $28(38)$ \\
\hline Weak & 9 (33) & $17(36)$ & $26(35)$ \\
\hline Absent & $12(45)$ & $8(17)$ & $20(27)$ \\
\hline Total & $27(100)$ & $47(100)$ & $74(100)$ \\
\hline \multicolumn{4}{|l|}{ MMP-2 } \\
\hline Strong & $21(78)$ & $42(91)$ & $63(85)$ \\
\hline Weak & $4(15)$ & $3(5)$ & $7(9)$ \\
\hline Absent & $2(7)$ & $2(4)$ & $4(6)$ \\
\hline \multirow{2}{*}{\multicolumn{4}{|c|}{ MMP-3 }} \\
\hline & & & \\
\hline Strong & $1(4)$ & $2(4)$ & $3(4)$ \\
\hline Weak & $4(15)$ & $13(28)$ & $17(23)$ \\
\hline Absent & $22(81)$ & $32(68)$ & $54(73)$ \\
\hline \multirow{2}{*}{\multicolumn{4}{|c|}{ MMP-9 }} \\
\hline & & & \\
\hline Strong & $8(30)$ & $22(47)$ & $30(41)$ \\
\hline Weak & $9(33)$ & $19(40)$ & $28(38)$ \\
\hline Absent & $10(37)$ & $6(13)$ & $16(21)$ \\
\hline Total & $27(100)$ & $47(100)$ & $74(100)$ \\
\hline \multicolumn{4}{|l|}{ TIMP-1 } \\
\hline Strong & $2(7)$ & $8(17)$ & $10(14)$ \\
\hline Weak & $7(26)$ & $13(28)$ & $20(27)$ \\
\hline Absent & $18(67)$ & $26(55)$ & $44(59)$ \\
\hline Total & $27(100)$ & $47(100)$ & $74(100)$ \\
\hline \multicolumn{4}{|l|}{ TIMP-2 } \\
\hline Strong & $5(19)$ & $7(16)$ & $12(16)$ \\
\hline Weak & $10(37)$ & $20(42)$ & $30(41)$ \\
\hline Absent & $12(44)$ & $20(42)$ & $32(43)$ \\
\hline Total & $27(100)$ & $47(100)$ & $74(100)$ \\
\hline
\end{tabular}

Results are expressed as number (\%).

Monoclonal antibodies to TIMP-1 and TIMP-2 were produced in a similar manner. The peptide sequences were FQALGDAADIR and DSGNDIYGNPI for TIMP-1 and TIMP-2 respectively. The peptides, each 11 residues long, corresponded to sequences specific for each of the TIMPs and were located in the $\mathrm{N}$ terminal domains of the TIMPs. Conjugation to carrier protein was by glutaraldehyde. ${ }^{24}$ Immunoblotting was performed as for the MMPs using $0.4 \mu \mathrm{g}$ of TIMP-1 or TIMP- 2 .
Isotyping of the monoclonal antibodies was performed with an Isostrip kit (Boehringer Mannheim, Lewes, Sussex), used according to the manufacturer's instructions.

IMMUNOHISTOCHEMISTRY

Sections of stomach tumours were immunostained with monoclonal antibodies to MMP-1 (clone 3B6), MMP-2 (clone 4D3), MMP-3 (clone 1B4), MMP-9 (clone 2C3), TIMP-1 (clone 2A5), and TIMP-2 (clone 3A4) using an alkaline phosphatase antialkaline phosphatase technique. ${ }^{28}$ Tissue sections were subjected to an antigen retrieval step by microwaving the sections for five minutes (MMP-2), seven minutes (TIMP-1, TIMP-2), or $10 \mathrm{~min}$ utes (MMP-1 and MMP-9) in $0.01 \mathrm{M}$ citrate buffer, $\mathrm{pH}$ 6.0, before application of the individual MMP or TIMP antibodies. All the primary antibodies were applied as undiluted tissue culture supernatant for 60 minutes at room temperature. The negative control in place of the primary monoclonal antibodies was $0.05 \mathrm{M}$ Tris buffered saline, $\mathrm{pH} 7.6$ (TBS) while the positive control for MMP-1, TIMP-1, and TIMP-2 was colonic adenocarcinoma and for MMP-2, MMP-3, and MMP-9 was lung containing intra-alveolar macrophages. Following incubation with the monoclonal antibodies, sections were sequentially incubated with rabbit antimouse immunoglobulin (1/100, Dako, High Wycombe, UK) and monoclonal alkaline phosphatase antialkaline phosphatase (1/100, Dako). After each antibody application, sections were washed in TBS for two successive five minute periods to remove unbound antibody. Alkaline phosphatase was determined using a substrate solution consisting of BCIP/NBT. After incubating the sections for 30 minutes at room

Table 2 The presence [number (percentage)] of individual MMPs and TIMPs in different stages of gastric cancer

\begin{tabular}{|c|c|c|c|c|c|c|}
\hline & \multicolumn{5}{|c|}{ Tumour stage } & \multirow[b]{2}{*}{ Total } \\
\hline & $1 a(n=5)$ & $1 b(n=17)$ & $2(n=43)$ & $3 a(n=8)$ & $4(n=1)$ & \\
\hline \multicolumn{7}{|l|}{ MMP-1 } \\
\hline Strong & $1(20)$ & $8(47)$ & $17(40)$ & $1(13)$ & $1(100)$ & $28(38)$ \\
\hline Weak & $1(20)$ & $5(29)$ & $16(37)$ & $4(50)$ & 0 & $26(35)$ \\
\hline Absent & $3(60)$ & $4(24)$ & $10(23)$ & $3(37)$ & 0 & $20(27)$ \\
\hline \multirow{2}{*}{\multicolumn{7}{|c|}{ MMP-2 }} \\
\hline & & & & & & \\
\hline Strong & $3(60)$ & $13(76)$ & $39(90)$ & $7(87)$ & $1(100)$ & $63(86)$ \\
\hline Weak & $1(20)$ & $3(18)$ & $2(5)$ & $1(13)$ & 0 & $7(9)$ \\
\hline Absent & $1(20)$ & $1(6)$ & $2(5)$ & 0 & 0 & $4(5)$ \\
\hline \multirow{2}{*}{\multicolumn{7}{|c|}{ MMP-3 }} \\
\hline & & & & & & \\
\hline Strong & 0 & 0 & $3(7)$ & 0 & 0 & $3(4)$ \\
\hline Weak & 0 & $4(24)$ & $9(21)$ & $4(50)$ & 0 & $17(23)$ \\
\hline Absent & $5(100)$ & $13(76)$ & $31(72)$ & $4(50)$ & $1(100)$ & $54(73)$ \\
\hline Total & $5(100)$ & $17(100)$ & $43(100)$ & $8(100)$ & $1(100)$ & $74(100)$ \\
\hline \multicolumn{7}{|l|}{ MMP-9 } \\
\hline Strong & $2(40)$ & $7(41)$ & $19(44)$ & $2(25)$ & 0 & $30(41)$ \\
\hline Weak & $2(40)$ & $7(41)$ & $15(35)$ & $3(37.5)$ & $1(100)$ & $28(38)$ \\
\hline Absent & $1(20)$ & $3(18)$ & $9(21)$ & $3(37.5)$ & 0 & $16(21)$ \\
\hline Total & $5(100)$ & $17(100)$ & $43(100)$ & $8(100)$ & $1(100)$ & $74(100)$ \\
\hline \multicolumn{7}{|l|}{ TIMP-1 } \\
\hline Strong & $1(20)$ & $2(12)$ & $7(16)$ & 0 & 0 & $10(14)$ \\
\hline Weak & $2(40)$ & $6(35)$ & $12(28)$ & 0 & 0 & $20(27)$ \\
\hline Absent & $2(40)$ & $9(53)$ & $24(56)$ & $8(100)$ & $1(100)$ & $44(59)$ \\
\hline \multirow{2}{*}{\multicolumn{7}{|c|}{ TIMP-2 }} \\
\hline & & & & & & \\
\hline Strong & $1(20)$ & $1(6)$ & $10(23)$ & 0 & 0 & $12(16)$ \\
\hline Weak & $1(20)$ & $7(41)$ & $16(37)$ & $5(62.5)$ & $1(100)$ & $30(41)$ \\
\hline Absent & $3(60)$ & $9(53)$ & $17(40)$ & $3(37.5)$ & 0 & $32(43)$ \\
\hline Total & $5(100)$ & $17(100)$ & $43(100)$ & $8(100)$ & $1(100)$ & $74(100)$ \\
\hline
\end{tabular}

Results are expressed as number (\%). 

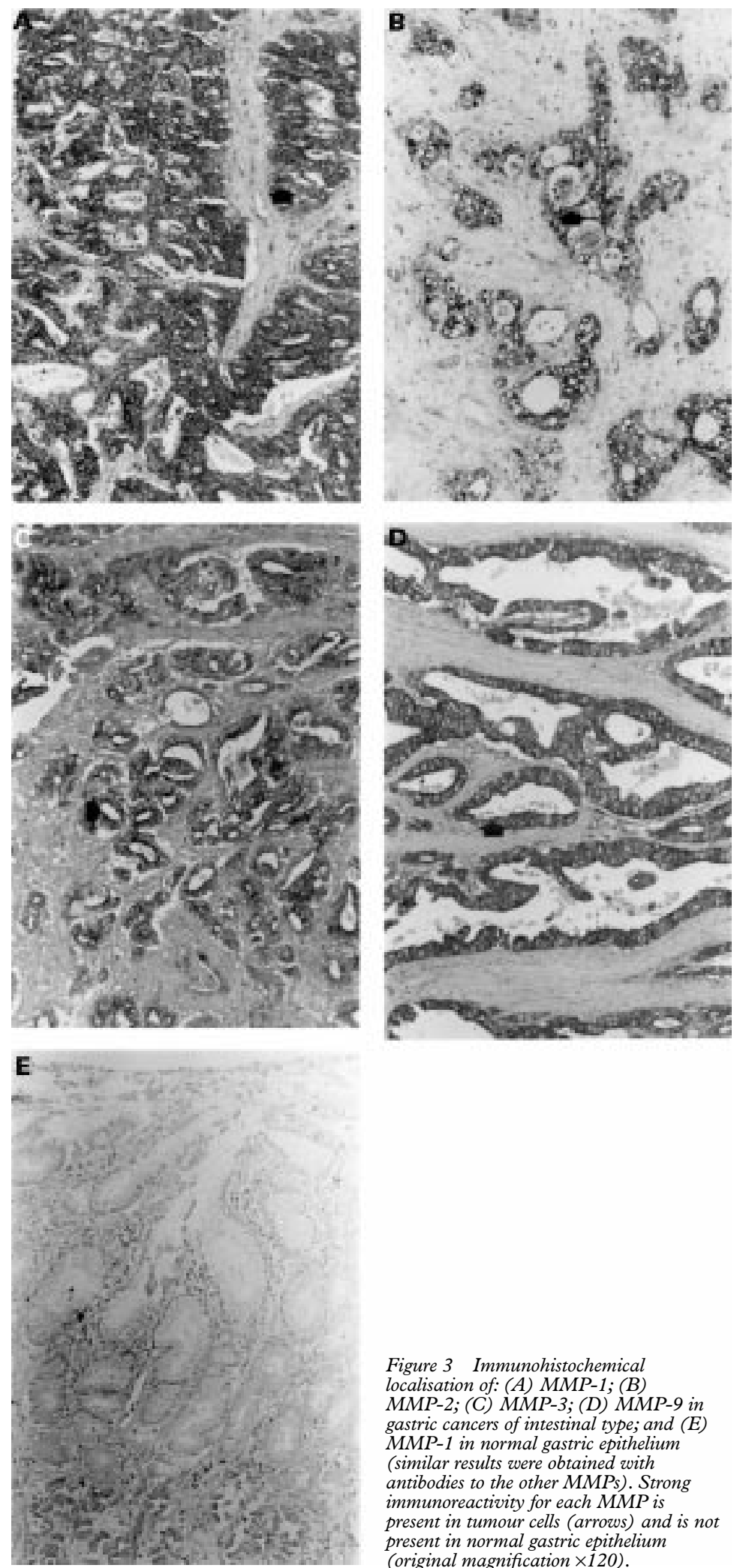

Figure 3 Immunohistochemical localisation of: (A) MMP-1; (B) $M M P-2 ;(C) M M P-3 ;$ (D) MMP-9 in gastric cancers of intestinal type; and $(E)$ $M M P-1$ in normal gastric epithelium (similar results were obtained with antibodies to the other $M M P s$ ). Strong immunoreactivity for each MMP is present in tumour cells (arrows) and is not present in normal gastric epithelium (original magnification $\times 120$ ).

temperature, the enzyme reaction was stopped by washing the sections for five minutes in hot tap water. The slides were then air dried and mounted in glycerine jelly.

When the immunohistochemistry was complete the sections were examined by light microscopy to determine the cellular localisation and distribution of immunostaining. The intensity of the immunostaining in different cell types was assessed as strongly positive, weakly positive, or absent (negative). A tumour was regarded as positive if any tumour cells showed immunostaining while a tumour was classified as negative if there was a complete absence of immunostaining in tumour cells as previously described. ${ }^{21}{ }^{22}$ The positive tumours were further divided into strongly positive and weakly positive groups and a tumour was regarded as strongly positive if any tumour cells showed strong immunoreactivity.

\section{STATISTICS}

Survival data were obtained after the assessment of the presence of MMPs and TIMPs in the stomach cancers was complete. Statistical analysis was performed using the computer program SPSS for Windows 95 (SPSS Inc., USA). Associations between MMPs or TIMPs, and histological type of tumour, lymph node status, or tumour stage were analysed using the $\chi^{2}$ test with Yates's correction. Cumulative patient survival was assessed by the method of Kaplan-Meier, and comparison of the MMP strongly positive, MMP weakly positive, and MMP negative survival curves was carried out using the log rank test as previously described. ${ }^{2}$

\section{Results}

MATRIX METALLOPROTEINASES

The immunoblots showed that the anti-MMP antibodies were specific for the appropriate MMP and did not cross react with other MMPs (fig 1). The antibodies recognised both the proenzyme and activated forms of the MMPs (fig 2). $\mathrm{N}$ terminal sequence analysis of the band at around $47 \mathrm{kDa}$ in the MMP-1 proenzyme lane indicated that it is a truncated form of the proenzyme lacking the first 64 residues and starting with Leu65. On treatment with trypsin and MMP-3, it is converted, like the intact proenzyme, to the activated form. The upper band in the activated MMP-2 lane is due to some residual unactivated proenzyme. The doublets evident in both the proenzyme and activated MMP-3 lanes can be explained by partial glycosylation as both components of the doublets had the same $\mathrm{N}$ termini. All the

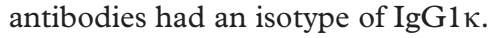

Immunohistochemistry of the sections of stomach cancer with the monoclonal antibodies to MMP-1, MMP-2, MMP-3, and MMP-9 showed cytoplasmic staining of tumour cells and there was no staining of normal gastric epithelium (fig 3). The staining for each MMP seemed to be uniform in all tumour cells with no apparent intratumour heterogeneity. Tables 1 and 2 summarise the presence of individual MMPs in different histological types of gastric cancer and different stages of gastric cancer. Almost three quarters $(73 \%, 54 / 74)$ of the tumours showed positive immunoreactivity for MMP-1. The intensity of immunostaining varied between tumours, with $38 \%(28 / 74)$ of cancers displaying strong staining in the tumour cells (fig 3 ) while $35 \%(26 / 74)$ of cases showed weak staining. MMP-1 was found mainly in tumours of intestinal type and less 


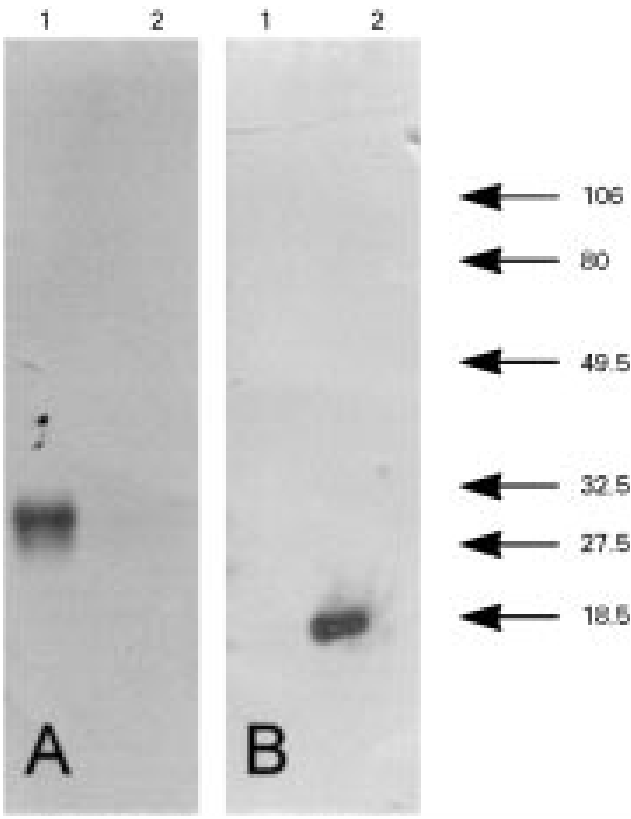

Figure 4 Immunoblot showing the specificity of the TIMP-1 and TIMP-2 antibodies. Section $A$ was immunostained with TIMP-1 antibody and section $B$ with TIMP-2 antibody. Lane 1, TIMP-1; lane 2, TIMP-2.

Molecular weight markers are shown on the right in $k D a$.

frequently in those of diffuse type $\left(\chi^{2}=7.55\right.$; $\mathrm{p}=0.02)$. MMP-1 immunoreactivity was also identified in fibroblasts. Nearly all the tumours (94\%, 70/74) showed positive immunostaining for MMP-2 with the majority $(85 \%, 63 / 74)$ of cancers displaying strong staining of tumour cells (fig 3) and only 9\% (7/74) of cases showing weak staining. Immunoreactivity of MMP-2 was also identified in macrophages. MMP-3 immunoreactivity was present in $27 \%(20 / 74)$ of

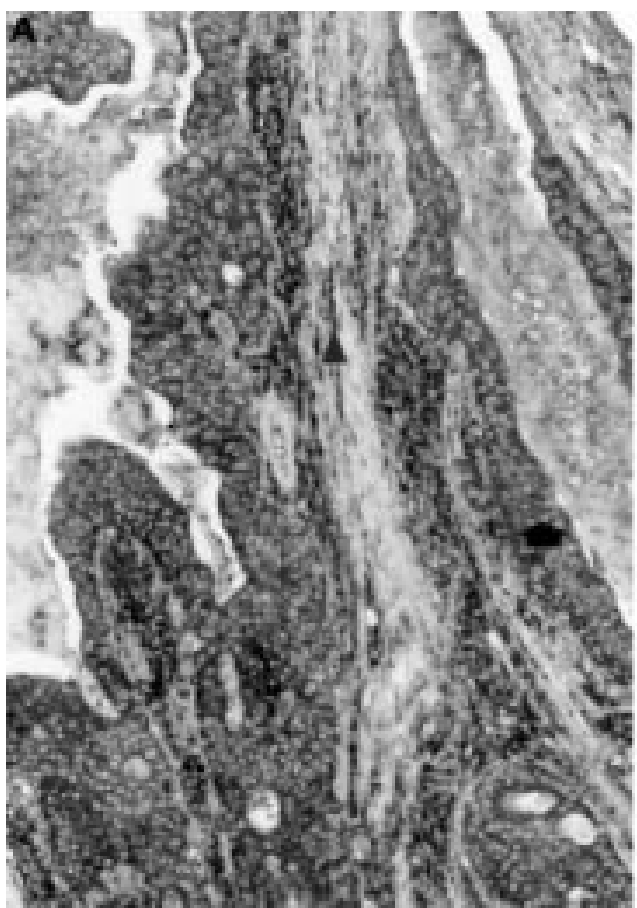

tumours with $4 \%$ (three) showing strong staining (fig 3) while 23\% (17) displayed weak immunoreactivity. Macrophages were consistently positive for MMP-3. MMP-9 immunoreactivity was present in $79 \%(58 / 74)$ of the gastric cancers, $41 \%(30 / 74)$ of the cases showing strong staining (fig 3) while weak staining was identified in a further $38 \%(28 / 74)$ of cases. One case showed weak immunoreactivity in both stromal and tumour cells. The presence of MMP-9 showed a highly significant correlation with MMP-1 ( $p=0.01$ ) and, as with MMP-1, the presence of MMP-9 was correlated with intestinal type tumours $\left(\chi^{2}=6.15, \mathrm{p}=0.04\right)$. There was no correlation between the presence of individual MMPs and tumour stage or lymph node status nor was the presence of individual MMPs related to survival.

TISSUE INHIBITORS OF MATRIX

METALLOPROTEINASES

The immunoblots showed that the TIMP-1 antibody recognised only TIMP-1 while the TIMP-2 antibody recognised only TIMP-2 (fig 4). The TIMP-1 and TIMP-2 antibodies

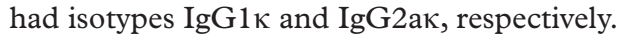

Positive immunoreactivity for both TIMPs was present in tumour cells and there was no immunoreactivity in normal gastric epithelium. Tables 1 and 2 summarise the presence of individual TIMPs in different histological types of gastric cancer and different stages of gastric cancer. TIMP-1 immunoreactivity was found in $41 \%(30 / 74)$ of the cases. Only $14 \%$ $(10 / 74)$ showed strong staining (fig 5), with $27 \%(20 / 74)$ displaying weak staining. TIMP-2 was found in just over half the cases $(57 \%$, $42 / 74)$. However, only $16 \%(12 / 74)$ of tumour samples were strongly stained (fig 5), with $41 \%$

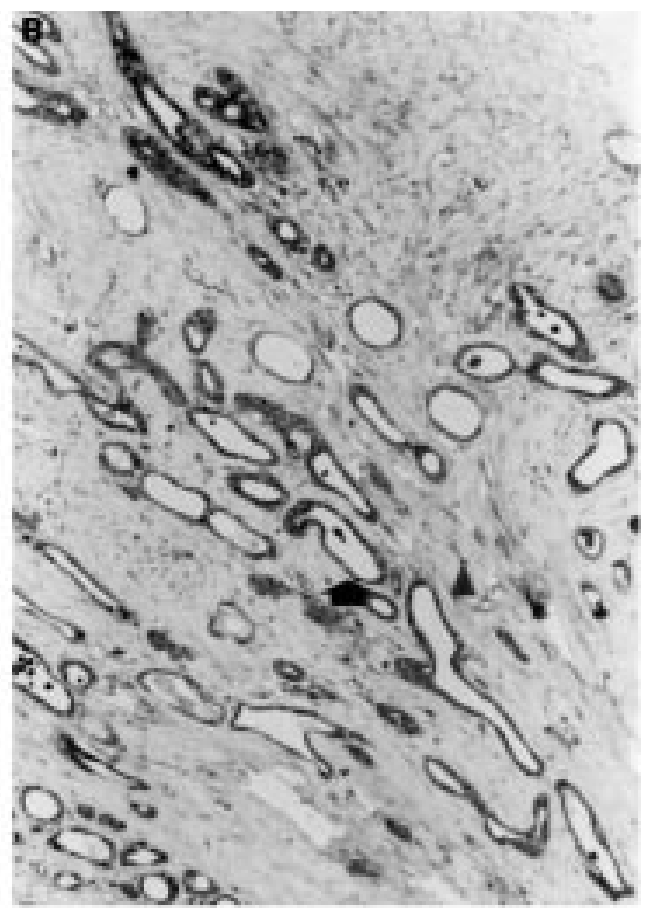

Figure 5 Immunohistochemical localisation of (A) TIMP-1 and (B) TIMP-2 in gastric cancers of intestinal type. TIMP-1 and TIMP-2 immunoreactivity is present in tumour cells (arrows). Immunostaining for TIMP-1 is also present in macrophages (arrowhead) and immunostaining for TIMP-2 is also present in fibroblasts (arrowhead) (original magnification $\times 120$ ). 
(30/74) showing weak staining. TIMP-1 immunoreactivity was also identified in macrophages while TIMP-2 immunoreactivity was present in fibroblasts. The presence of TIMP-1 and TIMP-2 did not correlate significantly with each other. The presence of TIMP-1 correlated with that of MMP-9 $(p=0.04)$. There were no correlations between the presence of TIMPs and tumour stage, histological type, lymph node status, or survival.

\section{Discussion}

The MMP and TIMP antibodies used in this study were raised using synthetic peptides corresponding to unique regions of each molecule. Because of the position of the epitopes recognised by the MMP antibodies on the $\mathrm{C}$ terminal domain of the individual MMPs the antibodies recognise both the latent and activated form of the individual MMP. In addition, these epitopes are remote from the active site which is involved with the interaction of MMPs with TIMPs, ${ }^{19}$ thus making it likely that binding of the MMP antibodies will not be inhibited by the interaction of TIMP with the MMP. The antibodies did not show any cross reaction and were all effective on formalin fixed, paraffin wax embedded sections which makes them very useful in diagnostic pathology.

In this study, MMP-1, MMP-2, MMP-3, and MMP-9, and their natural inhibitors TIMP-1 and TIMP-2 were examined in a series of 74 gastric cancers, most of which were advanced gastric cancers typical of those commonly found in the UK. The predominant tumour cell localisation of MMPs and TIMPs is consistent with other immunohistochemical studies of individual MMPs, especially MMP-2, and TIMPs in different types of malignant tumour. ${ }^{79-32} \mathrm{MMP}-2$ is considered to be important in tumour invasion, especially degradation of the basement membrane, ${ }^{11}$ and MMP-2 is frequently found in many types of malignant tumours. ${ }^{11}$ In the present study, MMP-2 was consistently localised to tumour cells and was not identified in adjacent normal gastric epithelium. The presence of MMP-2 in almost all the gastric cancers would support the importance of MMP-2 in tumour invasion and the expression of MMP-2 has been shown to be important in promoting invasion of gastric cancer cells in culture. ${ }^{33}$ In a recent study of stomach cancer in Dutch patients ${ }^{34}$ total MMP-2 concentration, which had been identified by gel zymography, was increased in cancer tissue compared with normal tissue and was associated with a relatively poor prognosis. Gel zymography, however, cannot provide information about the cellular localisation of MMPs. It also requires fresh tissue and is thus not practical for routine assessment of MMPs in diagnostic practice.

Our previous study has shown that MMP-1 is localised to tumour cells of colon ${ }^{21}$ and the presence of MMP-1 in these tumours is associated with poor prognosis: we have recently found essentially similar results with oesophageal tumours. ${ }^{22}$ However, we did not find an association between MMP-1 and survival in stomach cancer and further studies are required to establish the role of MMP-1 in tumour invasion and its relation to prognosis.

TIMP-1 and TIMP-2 were identified in $41 \%$ and $57 \%$ of tumours respectively and in both cases the TIMPs were confined in most instances to tumour cells. TIMP-2 is the natural inhibitor usually associated with MMP-2 while TIMP-1 is a more general inhibitor of the MMP family. TIMP-1 mRNA has previously been identified by reverse transcription polymerase chain reaction in five different cell lines derived from gastric cancers. ${ }^{33}$ Furthermore transfection of the complete human TIMP-1 cDNA into human gastric cancer cells notably decreased the formation of liver metastases of these cells when transplanted into nude mice. ${ }^{33}$

Low molecular weight broad spectrum inhibitors of $\mathrm{MMPs}^{36-38}$ are currently being developed for clinical use and it is important to identify those patients who are most likely to benefit from this type of therapy. Other strategies for the inhibition of individual MMPs are also under development including the use of ribozymes. ${ }^{39}$

We thank the Scottish Hospital Endowments Research Trust for financial support; Mr Bryan Dunbar and Mr Ian Davidson of the University of Aberdeen Protein Facility for amino acid sequence analysis and mass spectrometry; Dr Alan Galloway, British Biotech plc, for a gift of MMP-1, MMP-2, MMP-3, MMP-9, and TIMP-2; and Professor Gillian Murphy, University of East Anglia, for a gift of MMP-1 and TIMP-1.

1 Morson BC, Dawson IMP, Day DW, et al. Morson and Dawson's gastrointestinal pathology. 3rd edn. Oxford: Blackwell son's gastrointestinal patho

2 Hart IR, Saini A. Biology of tumour metastasis. Lancet 1992;339:1453-7.

3 Kohn EC, Liotta LA. Molecular insights into cancer invasion: strategies for prevention and intervention. Cancer Res 1995;55:1856-62.

4 Murphy G, Docherty AJP. The matrix metalloproteinases and their inhibitors. Am f Resp Cell Mol Biol 1992;7:120-5.

5 Stetler-Stevenson WG, Liotta LA, Kliener DE. Extracellular matrix 6: role of matrix metalloproteinases in tumor invasion and metastasis. FASEB $\mathcal{F}$ 1993;7:1434-41.

6 Davies B, Waxman J, Wasan H, et al. Levels of matrix metalloproteases in bladder cancer correlate with tumor grade and invasion. Cancer Res 1993;53:5365-9.

7 Boag AH, Young ID. Increased expression of the $72-\mathrm{kD}$ type IV collagenase in prostate adenocarcinoma. Am f Pathol IV collagenase in

8 Muller D, Wolf C, Abecassis J, et al. Increased stromelysin 3 gene expression is associated with increased local invasiveness in head and neck squamous cell carcinoma. Cancer Res 1993;53:165-9

9 Urbanski SJ, Edwards DR, Hershfield N, et al. Expression pattern of metalloproteinases and their inhibitors changes with the progression of human sporadic colorectal neoplasia. Diagn Mol Pathol 1993;2:81-9.

10 Woessner JF. Matrix metalloproteinases and their inhibitors in connective tissue remodelling. FASEB 7 1991;5:2145-54.

11 Tryggvason K, Höyhtä M, Pyke M. Type IV collagenases in invasive tumors. Breast Cancer Res Treat 1993;24:209-18.

Sato H, Seiki M. Membrane-type matrix metalloproteinases (MT-MMPs) in tumor metastasis. $f$ Biochem 1996;119: 209-15.

13 Sato H, Takino T, Okada Y, et al. A matrix metalloproteinase expressed on the surface of invasive tumour cells. ase expressed on the

14 Denhardt DT, Feng B, Edwards DR, et al. Tissue inhibitor of metalloproteinases (TIMP, akaEPA): structure, control of expression and biological functions. Pharmacol Ther 1993;59:329-41.

15 Cawston TE. Metalloproteinase inhibitors and the prevention of connective tissue breakdown. Pharmacol Ther 1996; 70:163-82.

16 Uría JA, Ferrando AA, Velasco G, et al. Structure and expression in breast tumors of human TIMP-3, a new member of the metalloproteinase inhibitor family. Cancer Res 1994;54:2091-4.

17 Greene J, Wang MS, Liu YLE, et al. Molecular cloning and characterization of human tissue inhibitor of metalloproteinase 4. F Biol Chem 1996;271:30375-80.

18 Douglas DA, Shi YE, Sang QA. Computational sequence analysis of the tissue inhibitor of metalloproteinase family. f Prot Chem 1997;16:237-55. 
19 Gomis-Ruth F-X, Maskos K, Betz M, et al. Mechanism of inhibition of the human matrix metalloproteinase inhibition of the human matrix metallopro

20 Albini A, Melchiori A, Santi L, et al. Tumor cell invasion $1991 ; 83: 775-9$.

21 Murray GI, Duncan ME, O'Neil P, et al. Matrix metalloproteinase-1 is associated with poor prognosis in colorectal cancer. Nat Med 1996;2:461-2.

22 Murray GI, Duncan ME, O'Neil P, et al. Matrix metalloproteinase-1 is associated with poor prognosis in oesophageal cancer. F Pathol 1998;185:256-61.

23 Sambrook J, Fritsch EF, Maniatis T. Molecular cloning: a laboratory manual. New York: Cold Spring Harbor Laboratory Press, 1989.

24 Hiraga A, Kemp, BE, Cohen P. Further studies on the structure of the glycogen-bound form of protein phosphatase-1 from rabbit skeletal muscle. Eur 7 Biochem 1987;163:253-8.

25 Duncan ME, McAleese SM, Booth NA, et al. A simple enzyme-linked immunosorbent assay (ELISA) for the neuron-specific $\gamma$ isozyme of human enolase (NSE) using neuron-specific $\gamma$ isozyme of human enolase (NSE) using
monoclonal antibodies raised against synthetic peptides monoclonal antibodies raised against synthetic peptides corresponding to isozym

26 Matsudaira P. Sequence from picomole of protein electroblotted onto polyvinylidene difluoride membranes. $7 \mathrm{Biol}$ Chem 1987;262:10035-8.

27 Murphy G, Docherty AJP, Hembry RM, et al. Research in arthritis: cellular events in osteoarthritis. $\mathrm{Br} \mathcal{F}$ Rheumatol 1991;30(suppl 1):25-31.

28 Murray GI, Duncan ME, Melvin WT, et al. Immunohistochemistry of neuron specific enolase with gamma-subunit specific anti-peptide monoclonal antibodies. $\mathcal{F}$ Clin Pathol 1993;46:993-6.

29 David L, Nesland JM, Holm R, et al. Expression of laminin, collagen IV, fibronectin and type IV collagenase in gastric carcinoma. Cancer 1994;73:518-27.
30 Nomura H, Fujimoto N, Seike M, et al. Enhanced production of matrix metalloproteinases and activation of matrix metalloproteinase 2 (gelatinase A) in human gastric carcinomas. Int f Cancer (Pred Oncol) 1996;69:9-16.

31 Kawano N, Osawa $\mathrm{H}$, Ito $\mathrm{T}$, et al. Expression of gelatinase A, tissue inhibitor of metalloproteinase-2, matrilysin, and trypsin(ogen) in lung neoplasms: an immunohistochemical study. Hum Pathol 1997;28:613-22.

32 Höyhtyä M, Fridman R, Komarek D, et al. Immunohistochemical localization of matrix metalloproteinase 2 and its
specific inhibitor TIMP-2 in neoplastic tissue with specific inhibitor TIMP-2 in neoplastic tissue
monoclonal antibodies. Int $\mathcal{F}$ Cancer 1994;56:500-5.

33 Schwarz G, Wang H, Lampen N, et al. Defining the invasive phenotype of proximal gastric cells. Cancer 1994;73:22-7.

34 Sier CFM, Kubben FJGM, Ganesh S, et al. Tissue levels of matrix metalloproteinases MMP-2 and MMP-9 are related to the overall survival of patients with gastric carcinoma. $\mathrm{Br}$ f Cancer 1996;74:413-17.

35 Watanabe $\mathrm{M}$, Takahashi $\mathrm{Y}$, Ohta $\mathrm{T}$, et al. Inhibition of metastasis in human gastric cancer cells transfected with tissue inhibitor of metalloproteinase 1 gene in nude mice. Cancer 1996;77(suppl):1676-80.

36 Watson SA, Morris TM, Robinson G, et al. Inhibition of organ invasion by the matrix metalloproteinase inhibitor batimastat (BB-94) in two human colon carcinoma metastasis models. Cancer Res 1995;55:3629-33.

37 Sledge GW, Qulali M, Goulet R, et al. Effects of matrix metalloproteinase inhibitor batimastat on breast cancer regrowth and metastasis in athymic mice. I Natl Cancer Inst 1995;87:1546-50.

38 Brown PD, Giavazzi R. Matrix metalloproteinase inhibition: a review of anti-tumour activity. Ann Oncol 1995;6:967-74.

39 Hua J, Muschel RJ. Inhibition of matrix metalloproteinase 9 expression by a ribozyme blocks metastasis in a rat sarcoma model system. Cancer Res 1996;56:5279-84. 